\title{
G-whizzes disagree over gravity
}

\section{Recent measurements of gravitational constant increase uncertainty over accepted value.}

The Newtonian constant of gravitation known in the finely tuned business of metrology as 'big $G$ ' - has come a long way since British physicist Henry Cavendish first measured the gravitational attraction of Earth in 1798. Although $G$ derived from Cavendish's measurements had an uncertainty of about $1 \%$, modern measurements have tightened that to just a couple of tens of parts per million.

But the relentless honing of $G$ may have hit a stumbling block. Two recent experiments are in striking disagreement with earlier findings, and the overall uncertainty in the value of the constant may be set to increase.

In Newton's equations of gravity, $G$ represents the size of the gravitational force. The constant is involved in the quest to unify the theories of gravity and quantum mechanics, and efforts to determine $G$ have contributed to progress in areas of experimental physics: elements of the apparatus first developed to measure the constant, for example, are now used in gravitational-wave detectors. But for some researchers, measuring $G$ is an end in itself. "It's the ultimate precision experiment," says James Faller, a physicist at the University of Colorado at Boulder.

Metrologists have traditionally measured $G$ using a torsion balance - a rod suspended from a wire. When masses are placed near the ends of the rod, their minute gravitational pull causes it to rotate by an amount proportional to G. In 2000, Jens Gundlach and Stephen Merkowitz at the University of Washington in Seattle used a new torsion-balance method to make the most precise measurement to date ${ }^{1}$ : $6.674215 \times 10^{-11}$ cubic metres per kilogram per square second, with an uncertainty of 14 parts per million (p.p.m.).

But that value is being challenged by two different methods that have now been developed to a level of precision comparable to Gundlach and Merkowitz's. In a paper accepted by Physical Review Letters ${ }^{2}$, Faller and Harold Parks at Sandia National Laboratories in Albuquerque, New Mexico, used a laser interferometer to measure the displacement of pendulum bobs by various masses. Their result $\left(6.67234 \times 10^{-11} \mathrm{~m}^{3} \mathrm{~kg}^{-1} \mathrm{~s}^{-2}\right.$, with an uncertainty of 21 p.p.m.) is an enormous 10 standard deviations lower than the value measured by Gundlach and Merkowitz. And in a paper ${ }^{3}$ published in 2009 in Physical Review Letters, researchers led by Jun Luo of Huazhong University of Science and Technology in Wuhan,

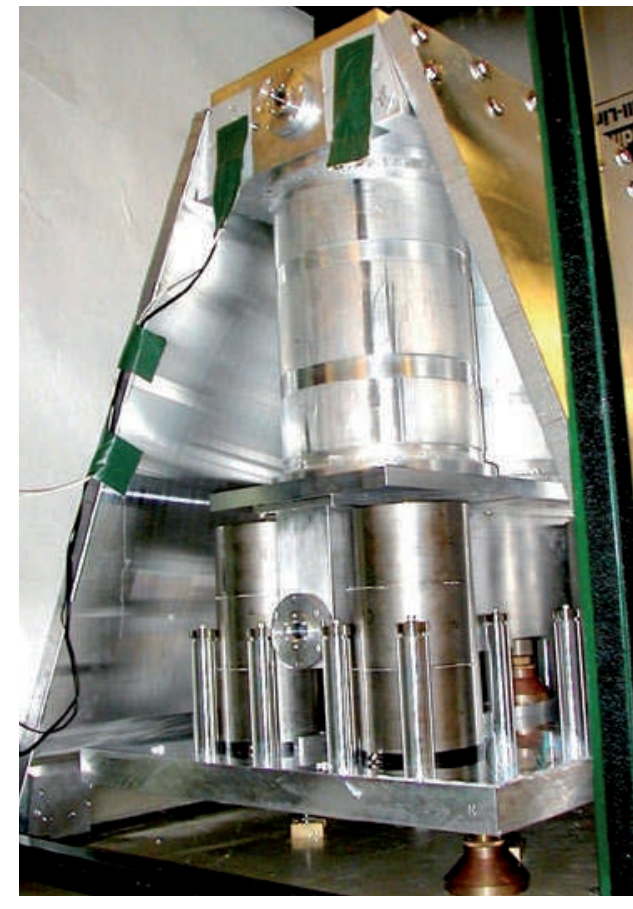

China, measured $G$ by comparing the time it took for a torsion pendulum to swing past masses placed at varying distances from it. They obtained a value of $6.67349 \times 10^{-11} \mathrm{~m}^{3} \mathrm{~kg}^{-1} \mathrm{~s}^{-2}$, with an uncertainty of 26 p.p.m., about three standard deviations below Gundlach and Merkowitz's value. Metrologists had expected much smaller disagreements among the results - probably no more than a couple of standard deviations.

Stephan Schlamminger of the University

\section{A CHANGING CONSTANT}

The recommended value of $\mathrm{G}$ - the gravitational constant - has risen slightly over the past four decades. But the latest measurements will probably cause a downward revision.

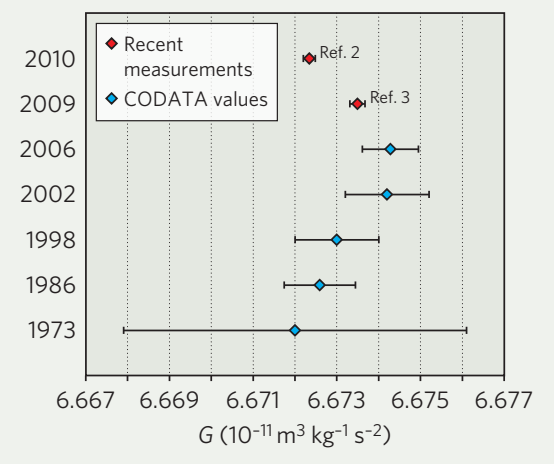

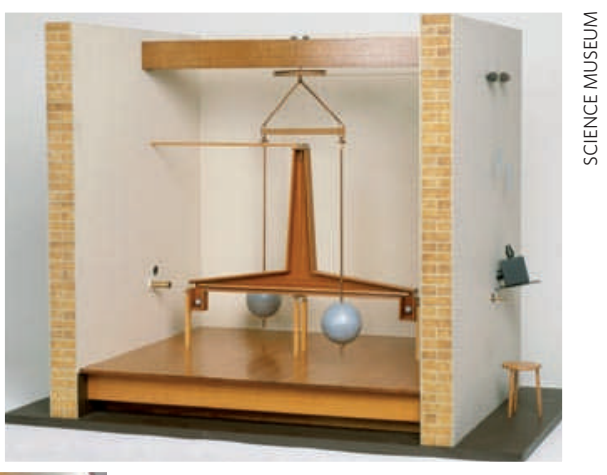

A model of Henry Cavendish's torsion balance (above) and its latest successor, a laser interferometer.

of Washington, who measured $G$ while at the University of Zurich in Switzerland and reported ${ }^{4}$ a result consistent with that of Gundlach and Merkowitz, says he cannot explain the inconsistency. It may be down to systematic error, which is why it is so important to measure $G$ in a variety of ways, he says. "People are obviously overlooking effects and not taking that into account in their experiments," adds Barry Taylor of the National Institute of Standards and Technology in Gaithersburg, Maryland.

The latest results mean that the Paris-based Committee on Data for Science and Technology (CODATA), which recommends values of physical constants every four years (see 'A changing constant'), will probably revise $G$ in its next set of values, finalized in early 2011. "Those new values will pull the value down," says Taylor, who sits on the committee. "The final uncertainty will be the same or larger."

Faller says the fear of being wrong can cause investigators to wait many years before publishing results that don't agree with previous measurements. He and Parks ran their experiment in 2004, and have spent the time since then searching for effects they might have missed. But he's sure their measurement is sound: "I feel like I've checked everything and I have to wash my hands."

Eugenie Samuel Reich

1. Gundlach, J. H. \& Merkowitz, S. M. Phys. Rev. Lett. 85, 2869 (2000).

2. Parks, H. V. \& Faller, J. E. Phys. Rev. Lett. http://xxx.lanl.gov/ abs/1008.3203 (2010)

3. Luo, J. et al. Phys. Rev. Lett. 102, 240801 (2009).

4. Schlamminger, S. et al. Phys. Rev. D 74, 082001 (2006). 